\title{
CSF total and oligomeric a-Synuclein along with TNF-a as risk biomarkers for Parkinson's disease: a study in LRRK2 mutation carriers
}

Nour K. Majbour ${ }^{1}$, Jan O. Aasly ${ }^{2,3}$, Eldbjørg Hustad ${ }^{2,3}$, Mercy A. Thomas', Nishant N. Vaikath ${ }^{1}$, Naser Elkum, Wilma D. J. van de Berg ${ }^{5}$, Takahiko Tokuda ${ }^{6}$, Brit Mollenhauer ${ }^{7}$, Henk W. Berendse ${ }^{8}$ and Omar M. A. El-Agnaf ${ }^{{ }^{*}}$

\begin{abstract}
Background: Asymptomatic carriers of leucine-rich repeat kinase 2 (LRRK2) gene mutations constitute an ideal population for discovering prodromal biomarkers of Parkinson's disease (PD). In this study, we aim to identify CSF candidate risk biomarkers of PD in individuals with LRRK2 mutation carriers.

Methods: We measured the levels of CSF total- (t-), oligomeric (o-) and phosphorylated S129 (pS129-) a-syn, totaltau (tTau), phosphorylated threonine 181 tau (pTau), amyloid-beta 40 (A $\beta-40)$, amyloid-beta-42 (A $\beta-42)$ and 40 inflammatory chemokines in symptomatic $(n=23)$ and asymptomatic $(n=51)$ LRRK2 mutation carriers, subjects with a clinical diagnosis of PD $(n=60)$ and age-matched healthy controls $(n=34)$. General linear models corrected for age and gender were performed to assess differences in CSF biomarkers between the groups. Markers that varied significantly between the groups were then analyzed using backward-elimination logistic regression analysis to identify an ideal biomarkers panel of prodromal PD.
\end{abstract}

Results: Discriminant function analysis revealed low levels of CSF t-a-syn, high levels of CSF o-a-syn and TNF-a best discriminated asymptomatic LRRK2 mutation carriers from both symptomatic PD and healthy controls. Assessing the discriminative power using receiver operating curve analysis, an area under the curve $>0.80$ was generated.

Conclusions: The current study suggests that CSF t-, o-a-syn and TNF-a are candidate risk biomarkers for the detection of PD at the prodromal stage. Our findings also highlight the dynamic interrelationships between CSF proteins and the importance of using a biomarkers' panel approach for an accurate and timely diagnosis of PD.

Keywords: Parkinson's disease, LRRK2 mutation carriers, Alpha-synuclein oligomers, Biomarkers, Inflammatory markers

\section{Background}

Our understanding of the genetic basis of Parkinson's disease (PD) has increased tremendously over the past 20 years. Mutations in the gene encoding alpha-synuclein $(\alpha-$ syn) were the first to be associated with genetic PD. Another monogenic causative factor in PD patients is

\footnotetext{
* Correspondence: oelagnaf@qf.org.qa

${ }^{1}$ Neurological Disorders Research Center, Qatar Biomedical Research Institute, Hamad Bin Khalifa University, Qatar Foundation, P.O. Box 5825, Doha, Qatar Full list of author information is available at the end of the article
}

leucine-rich repeat kinase 2 (LRRK2), of which more than 100 variants have been identified [1]. Asymptomatic carriers of $L R R K 2$ mutations constitute an ideal population for identifying predictive biomarkers of $P D$ for several reasons: 1) a high risk of conversion to PD, 2) dopaminergic neuronal loss demonstrated by positron emission tomography (PET) scanning, and 3) similarity of the clinical phenotype of LRRK2-associated PD to that of patients with sporadic PD (sPD). While the exact involvement of LRRK2 in PD pathogenesis remains only partially 
understood, converging evidence suggests a role for LRRK2 in modulating inflammation [2, 3]. As PD has been proposed to start as an inflammatory disease [4, 5], it is plausible to suggest that there may be a link between LRRK2 mutations and inflammation.

Several research groups, including ours, have explored the potential of CSF alpha-synuclein ( $\alpha$-syn) forms as diagnostic or progression biomarkers for PD. Total $\alpha$-syn (t- $\alpha$-syn) levels were reported to be lower in PD, whereas oligomeric (o- $\alpha$-syn) and phosphorylated Ser129- $\alpha$-syn (pS129- $\alpha$-syn) appear to be elevated [6-9]. CSF core biomarkers of Alzheimer's disease (AD) pathology have also been widely explored in PD cases. While a drop in CSF Amyloid-beta $(\mathrm{A} \beta-42)$ levels have been reported in $\mathrm{PD}$ [10], the biomarker profile of total tau (tTau), and phosphorylated threonine 181 tau (pTau) were variable [11, 12]. More importantly, the potential of the aforementioned proteins as markers for PD at preclinical stage remains largely unexplored. Carriers of LRRK2 mutations have an elevated risk of developing PD and they therefore represent a useful population in which to identify biomarkers of prodromal PD [13]. However, there is a paucity of data on different forms of $\alpha$-syn, AD-related proteins and inflammatory biomarkers in LRRK2 mutation carriers [14-16]. In the present study, our primary objective was to identify a panel of CSF biomarkers for the early detection of PD, preferably at the presymptomatic stage. A secondary objective was to study whether CSF levels of particular biomarkers were associated with severity of clinical symptoms of PD. Towards that end, we measured the levels of different $\alpha$-syn species, AD-related proteins and 40 different inflammatory markers in CSF samples from a well-characterized Norwegian cohort of 74 subjects with LRRK2 mutations: 23 symptomatic individuals and 51 asymptomatic mutation carriers. In parallel, we included 60 patients with sporadic (i.e. idiopathic) PD (sPD) and 43 healthy control subjects (first-degree relatives of LRRK2 mutation carriers (Ctrl)).

\section{Methods}

\section{Patient selection and CSF sampling}

Patient selection criteria and the method of CSF collection were as described in previous publications $[16,17]$. In total, 74 Norwegian individuals from 12 different families with LRRK2 mutations were assessed in the current study. Twenty-three patients were clinically diagnosed with $\mathrm{PD}$, whereas 51 patients were healthy, asymptomatic LRRK2 mutation carriers when enrolled in the study. These families have been extensively described in previous reports [17-19]. In addition, 60 patients with sPD and 43 age-matched controls were recruited for this study from St. Olav's Hospital at the University Hospital of Trondheim in Norway. The control group was composed of first-degree relatives of LRRK2 mutation carriers who were not carrying LRRK2 mutations. PD clinical diagnoses were made by experienced senior clinicians based on guidelines described by Gelb and colleagues [20] and disease stage was assessed according to the Hoehn and Yahr (H\&Y) scale. All patients with sPD were screened and confirmed to be negative for known LRRK2 mutations. Patients with an age at onset of $\leq 50$ years were confirmed to be negative for known pathogenic mutations in Parkin and PINK1. All family members of LRRK2 patients were examined for clinical features of PD by movement disorder specialists and found to be asymptomatic, although a few had mild premotor signs and an increased Unified Parkinson's Disease Rating Scale (UPDRS) score (three cases scored $>10$ on UPDRS-III) [21]. The LRRK2-mutant PD patients were taking levodopa, and some were taking dopamine agonists and/or monoamine oxidase-B (MAO-B) inhibitors.

To collect CSF, lumbar punctures were performed in overnight fasted patients between 8 and $10 \mathrm{am}$. CSF samples were aliquoted in $1.2-1.5 \mathrm{ml}$ low-binding tubes, and one vial was sent for routine laboratory analysis (i.e., white and red blood cell count, total protein and glucose levels, according to the Parkinson's Progression Markers Initiative [PPMI] protocol), whereas the majority of the vials were frozen fewer than $15 \mathrm{~min}$ after collection following centrifugation at $2000 \mathrm{~g}$ at $4{ }^{\circ} \mathrm{C}$ then subaliquoted and stored at $-80^{\circ} \mathrm{C}$ until further analysis. All patients provided signed informed consent, and the study was approved by the Regional Committee for Medical and Health Research Ethics.

\section{Measurement of alpha-synuclein species}

All immunoassays used to measure the different species of $\alpha$-syn were developed in-house and described in previous reports $[9,22,23]$. Briefly, to capture t- or pS129$\alpha$-syn, a 384-well ELISA microplate was coated with 0.1 or $0.5 \mu \mathrm{g} / \mathrm{ml}$ Syn-140 (a sheep anti- $\alpha$-syn polyclonal antibody) in $200 \mathrm{mM} \mathrm{NaHCO}$, pH $9.6(50 \mu \mathrm{l} /$ well $)$ by overnight incubation at $4{ }^{\circ} \mathrm{C}$, while $0.2 \mu \mathrm{g} / \mathrm{ml}$ of our mouse conformation-specific antibody, Syn-O2, was used to capture o- $\alpha$-syn. After incubation with $100 \mu \mathrm{l} /$ well of blocking buffer (PBS-T containing 2.25\% gelatin) for $2 \mathrm{~h}$ at $37^{\circ} \mathrm{C}, 50 \mu \mathrm{l} /$ well of the CSF samples (diluted 1:2 in artificial CSF) along with serial dilutions of recombinant human $\mathrm{t}$-, pS129-or o- $\alpha$-syn $(50 \mu \mathrm{l})$ were dispensed in each well, and the plate was incubated at $37^{\circ} \mathrm{C}$ for $2.5 \mathrm{~h}$. After washing with PBS-T, $50 \mu \mathrm{l} /$ well of 11D12 (a mouse anti- $\alpha$-syn monoclonal antibody) [9], PS129 (a mouse anti-pS129- $\alpha$-syn monoclonal antibody) [9], or FL-140 (rabbit polyclonal antibody, Santa Cruz Biotechnology, Santa Cruz, CA, USA), for measuring t-, pS129- $\alpha$-syn, or $\mathrm{o}-$, respectively, were added to the corresponding wells and incubated at $37^{\circ} \mathrm{C}$ for $2 \mathrm{~h}$. Next, the plates were washed and incubated for $2 \mathrm{~h}$ at $37^{\circ} \mathrm{C}$ with $50 \mu \mathrm{l} /$ well of 
species-appropriate secondary antibody (goat anti-rabbit IgG HRP or donkey anti-mouse IgG HRP, Jackson ImmunoResearch Laboratories Inc., USA, 1:20,000 dilution). After washing, the plates were incubated with $50 \mu \mathrm{l} /$ well of enhanced chemiluminescent substrate (Super Signal ELISA Femto, Pierce Biotechnology, USA). The chemiluminescence, expressed in relative light units, was immediately measured using a PerkinElmer Envision multi-label plate reader (PerkinElmer, Finland). CSF samples were measured in a blinded fashion and randomized for analysis, with all LRRK2 symptomatic/ asymptomatic, $\mathrm{PD}$ and $\mathrm{HC}$ samples being tested together on the same ELISA microplates. A series of internal controls was also run to check for run-to-run variations. The concentrations of $\alpha$-syn species in the samples were calculated using the corresponding standard curves.

\section{Measurement of AD biomarkers}

CSF $A \beta 42, A \beta 40$, total tau (tTau), and phosphorylated threonine 181 tau (pTau) were measured using MILLIPLEX ${ }^{\oplus}$ MAP Human Amyloid Beta Tau Magnetic Bead Panel (Luminex xMAP) run on the Bio-Plex 3D instrument (Bio-Rad Laboratories, Hercules, CA) according to manufacturer's instructions. The kit allows simultaneous quantification of $\mathrm{A} \beta 40, \mathrm{~A} \beta 42$, tTau, and $\mathrm{pTau}$.

\section{Measurement of inflammatory markers}

A magnetic human chemokine bioplex assay (Bio-Rad Laboratories, Hercules, CA) was used to measure 40 chemokines from human CSF samples (6Ckine/CCL21, BCA-1/ CXCL13, CTACK/CCL27, ENA-78/CXCL5, Eotaxin/ CCL11, Eotaxin-2/CCL24, Eotaxin-3/CCL26, Fractalkine /CX3CL1, GCP-2/CXCL6,GM-CSF, Gro- $\alpha / C X C L 1$, Gro$\beta / C X C L 2$, I-309/CCL1, IFN- $\Upsilon$, IL-1 $\beta$, IL-2, IL-4, IL-6, IL8/CXCL8, IL-10, IL-16, IP-10/CXCL10, I-TAC/CXCL11, MCP-1/CCL2, MCP-2/CCL8, MCP-3/CCL7, MCP-4/ CCL13, MDC/CCL22, MIF, MIG/CXCL9, MIP-1 $\alpha / C C L 3$, MIP-1 $\delta / C C L 15$, MIP-3 $\alpha / C C L 20, M I P-3 \beta / C C L 19$, MPIF1/CCL23, SCYB16/CXCL16, SDF- $\alpha+\beta / C X C L 12$, TAR C/CCL17, TECK/CCL25, TNF- $\alpha$ ). Bioplex assays was run according to the manufacturer's instructions using the recommended 1 in 2 dilution of CSF. Plates were read using the Bio-Plex ${ }^{\circ}$ 3D suspension array system; the next generation multiplexing platform based on xMAP technology. Enclosed standards were used to generate an 8point standard curve to which a 5-parameter logistic curve was fitted and were used to quantify unknown concentrations using BioPlex manger software. The coefficient of variance between duplicates was mostly $<10 \%$, as was the variance between standard curves run on separate plates. Only chemokines with robust readings above background were considered for further analysis.

\section{Statistical analysis}

IBM SPSS software (version 24.0, Chicago, IL, USA) was used for the statistical analyses of the data, whereas $R$ 3.6.1 was used for plotting the box and whisker plots.

Demographic and clinical characteristics were compared between study groups using chi-square tests, analysis of variance with post hoc Bonferroni tests or Kruskal- Wallis tests followed by Mann-Whitney U tests, where appropriate. All datasets were tested for normality and the presence of outliers. As data were considered inappropriate for parametric analyses, Spearman rankorder correlation coefficients were used to examine correlations within the study group. For all CSF biomarkers that showed robust readings above background (i.e. $\alpha$ syn species, AD markers and 18 inflammatory markers), differences between the diagnostic groups were assessed using general linear models (GLMs) corrected for age and gender with post-hoc Bonferroni corrections for multiple comparisons. Only biomarkers that varied significantly among the study groups were included in the next Discriminant Function analyses. Since our main objective was to identify predictive markers for the early detection of PD, both symptomatic LRRK2 mutation carriers and SPD groups were combined for discriminant function analysis. Discriminant function analysis evaluates canonical discriminant functions based on combinations of the selected markers which contribute maximally to group separation and assesses how well these canonical discriminant functions discriminate the diagnostic groups. CSF biomarker data were Z-transformed prior to discriminant function analysis.

We also performed multivariate logistic regression analyses with backward stepwise selection (separate analyses for each comparison). The control group was entered as a reference category and $\mathrm{t}$-, $\mathrm{o}$ - and pSer129- $\alpha$ syn, TNF- $\alpha$ and IL-16 were enrolled as predictors. For the resulting models, we report AUC, sensitivity, specificity and OR $(95 \% \mathrm{CI})$ of the individual model.

\section{Results \\ Patient population and demographics}

Demographics, clinical characteristics and CSF biomarkers levels of the study groups are summarized in Table 1. Twenty-three of the 74 subjects $(30 \%)$ with LRRK2 mutations analyzed had a manifest PD and were carrying either the most common LRRK2 mutation, G2019S, or a different LRRK2 mutation, N1437H. The symptomatic $L R R K 2$ mutation carriers had a mean age of $60 \pm 11$ years. 51 subjects of the 74 subjects (70\%) had no symptoms of PD at the time of CSF sample collection and these cases had a mean age of $57 \pm 14$ years. There was no significant difference in disease duration between the symptomatic LRRK2 mutation carriers and the sPD patients. Moreover, no difference was found between 
Table 1 Demographics and CSF biomarkers by diagnostic group

\begin{tabular}{|c|c|c|c|c|}
\hline & Ctrl $(n=43)$ & $\mathrm{sPD}(n=60)$ & $\begin{array}{l}\text { Asymptomatic } L R R K 2 \\
\text { mutation carriers }(n=51)\end{array}$ & $\begin{array}{l}\text { Symptomatic } \angle R R K 2 \\
\text { mutation carriers }(n=23)\end{array}$ \\
\hline Age $(y)$, mean $\pm S D^{a}$ & $49 \pm 18$ & $57 \pm 10$ & $57 \pm 14$ & $60 \pm 11$ \\
\hline Gender (male), $\mathrm{n}(\%)^{\mathrm{a}}$ & 19 (38.0\%) & $36(52.9 \%)$ & $26(50 \%)$ & $6(25 \%)$ \\
\hline Disease duration $(y)^{b}$ & NA & $4(1-6)$ & NA & $7(5-19)$ \\
\hline $\mathrm{MoCA}^{\mathrm{c}}$ & $27(27-29)$ & $27(25-28)$ & $27(26-28)$ & $25(23.7-27)$ \\
\hline $\mathrm{H} \& \mathrm{Y}$ & NA & $2(2-2)$ & $0(0-0)$ & $2(2-3)$ \\
\hline UPDRS-III & NA & $24(19-29)$ & $3(0.25-5)$ & $24(19.5-27)$ \\
\hline t-a-syn $(p g / m L)^{e}$ & 816 (596-1112) & $573(466-710)^{* * *}$ & $617(431-803)^{* *}$ & $608(432-740)^{* *}$ \\
\hline o-a-syn $(p g / m L)^{e}$ & $161(148-186)$ & $187.5(170.5-219.8)^{* *}$ & $183(160-230)^{* *}$ & $182(146-196)$ \\
\hline pS129-a-syn $(p g / m L)^{e}$ & $116(103-145)$ & $139(114.25-163)$ & $121(94-150)$ & $122(106-145)$ \\
\hline tTau $(\mathrm{pg} / \mathrm{mL})^{\mathrm{f}}$ & $141.2(89.6-201.3)$ & $134.7(88.3-224.9)$ & $123.6(86.7-236.4)$ & $113.5(94.9-167.8)$ \\
\hline pTau $(p g / m L)^{f}$ & $12(9-16)$ & $14.3(9.6-21.7)$ & $14.7(10.7-20.8)$ & $15.9(10.5-22.4)$ \\
\hline$A \beta-40(p g / m L)^{f}$ & $2506(1967-3120)$ & 2595.7 (2193.7-3098.2) & $2776.2(2287.6-4020.6)$ & 3048.9 (1959.3-3810.8) \\
\hline$A \beta-42(p g / m L)^{f}$ & $517.9(404.77-738)$ & $535.4(405.11-662.6)$ & $580.3(457.8-905.3)$ & 539.9 (410.9-880.9) \\
\hline $\operatorname{IL}-16^{9}$ & $6.5(4.9-8)$ & $5.1(4-6.6)^{*}$ & $5.1(3.9-6.8)$ & $4.3(2.8-5.3)$ \\
\hline TNF- $a^{g}$ & $3.3(2.6-5.5)$ & $4.2(2.4-6.5)$ & $5.5(4.2-7.4)^{*}$ & $5.5(2.5-7.3)$ \\
\hline
\end{tabular}

Data are expressed as mean $\pm S D$, median (IQR), or $n(\%)$. Demographical differences between groups were analyzed using analysis of variance with post hoc Bonferroni tests (age), X2 tests (gender), and Kruskal-Wallis with post hoc Mann-Whitney $U$ tests (MoCA, H\&Y, UPDRS and disease duration). Differences in CSF biomarker levels between groups were assessed with a GLM adjusted for age and gender. T-, o-, and pS129- $\alpha-5 y n$, IL-16, TNF- $\alpha$, tTau, ptau, A 340 and A $\beta-42$ were log-transformed, yet presented as raw data $\left({ }^{*} p<0.05,{ }^{* *} p<0.01,{ }^{* * *} p<0.001\right.$ compared with Ctrl)

A 1 1-42, amyloid $\beta 1-42$; Ctrl, healthy controls; H\&Y, Hoehn and Yahr scale; IL-16, interleukin-16; MoCA, Montreal Cognitive Assessment; NA, not applicable; o-a-syn, oligomeric a-synuclein; pSer129-a-synuclein, phosphorylated a-synuclein protein at serine 129; pTau, tau phosphorylated at threonine 181; sPD, sporadic PD; TNFa, tumor necrosis factor-alpha; tTau, total tau protein; t-a-syn, total a-synuclein; UPDRS-III, Unified Parkinson's Disease Rating Scale

${ }^{a} \mathrm{C}$ trl, $n=43$; sPD, $n=58$; Asymptomatic LRRK2 mutation carriers, $n=51$, Symptomatic LRRK2 mutation carriers, $n=23$

${ }^{b} \mathrm{Ctrl}, \mathrm{n}=\mathrm{NA}$; sPD, $n=59$; Symptomatic LRRK2 mutation carriers, $n=21$

c Ctrl, $n=15$; sPD, $n=59$; Asymptomatic LRRK2 mutation carriers, $n=48$, Symptomatic LRRK2 mutation carriers, $n=22$

${ }^{d}$ Ctrl, $n=15$; sPD, $n=59$; Asymptomatic LRRK2 mutation carriers, $n=48$, Symptomatic LRRK2 mutation carriers, $n=21$

e Ctrl, $n=43$; sPD, $n=60$; Asymptomatic LRRK2 mutation carriers, $n=51$, Symptomatic LRRK2 mutation carriers, $n=23$

${ }^{f} \mathrm{Ctrl}, n=28$; SPD, $n=40$; Asymptomatic LRRK2 mutation carriers, $n=26$, Symptomatic LRRK2 mutation carriers, $n=11$

${ }^{9} \mathrm{Ctrl}, n=28$; sPD, $n=42$; Asymptomatic LRRK2 mutation carriers, $n=24$, Symptomatic LRRK2 mutation carriers, $n=12$

groups with regard to the routine CSF levels of whitered cell count as well as the total protein, albumin, and glucose levels, including the plasma glucose level. Controlling for age and gender did not significantly alter the results.

\section{CSF biomarkers levels in diagnostic groups}

The levels of CSF t-, o- and pS129- $\alpha$-syn in the different diagnostic groups are presented in Table 1 and Fig. 1. The GLM showed differences between diagnostic groups in CSF t-, o- and pS129- $\alpha$-syn, TNF- $\alpha$ and IL-16 levels $(P<0.01$, adjusted for age and gender; Table 1$)$. Post-hoc tests revealed that in comparison to Ctrl (median and $\mathrm{IQR}=816(596-1112) \mathrm{pg} / \mathrm{ml}, n=43)$, levels of CSF $\mathrm{t}-\alpha-$ syn were significantly lower in SPD group (median and $\mathrm{IQR}=573 \quad(466.5-710.5), n=60) \quad(P<0.001, \quad$ Fig. $1 \mathrm{a})$, asymptomatic LRRK2 mutation carriers (median and $\mathrm{IQR}=617(431-803) \mathrm{pg} / \mathrm{ml}, n=51)(P<0.01$, Fig. 1a), and symptomatic LRRK2 mutation carriers (median and $\mathrm{IQR}=608(432-740) \mathrm{pg} / \mathrm{ml}, n=23) \quad(P<0.01$, Fig. 1a). There were no significant differences in the levels of CSF $t-\alpha$-syn between the groups of sPD, symptomatic and asymptomatic LRRK2 mutation carriers. On the other hand, and as shown in Fig. 1b, both SPD (median and IQR $=187.5(170.5-219.8) \mathrm{pg} / \mathrm{ml}, n=60)(P<0.001$, Fig. 1b) and asymptomatic LRRK2 (median and IQR = $183(160-230) \mathrm{pg} / \mathrm{ml}, n=51)(P<0.01$, Fig. 1b) groups had CSF profile with higher levels of o- $\alpha$-syn compared with Ctrl (median and IQR $=161(148-186) \mathrm{pg} / \mathrm{ml}, n=$ 43). Examination of CSF levels of pS129- $\alpha$-syn revealed a trend of an increase in SPD group (median and IQR = $139(114.25-163) \mathrm{pg} / \mathrm{ml}, \mathrm{n}=60)$ compared to Ctrl (median and $\mathrm{IQR}=116(103-145) \mathrm{pg} / \mathrm{ml}, n=43)$, however the difference did not reach statistical significance. (Fig. 1c). The ratios of o- $\alpha$-syn/t- $\alpha$-syn $\%$ and pSer129- $\alpha$-syn/ $\mathrm{t}-\alpha$-syn were both higher in SPD, symptomatic and asymptomatic LRRK2 mutation carriers compared with Ctrl $(P<0.01$; Fig. 1d, e).

The age- and gender-adjusted GLM revealed no significant differences in levels of AD biomarkers between the study groups.

To determine whether inflammatory biomarkers were altered among the different study groups, a panel of 40 proinflammatory cytokines, chemokines, and growth 

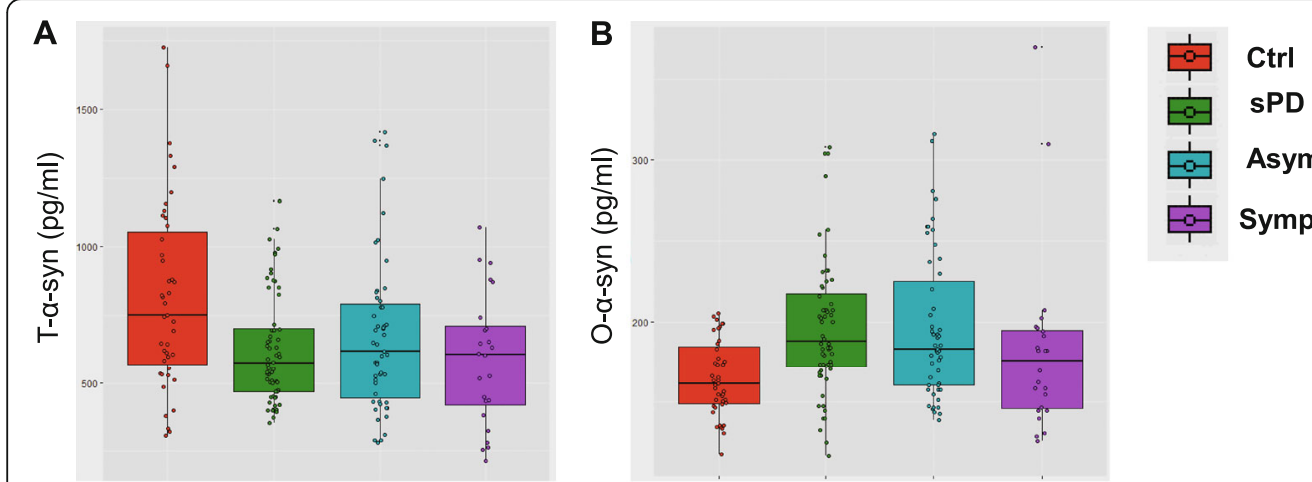

\section{Asymptomatic LRRK2 carriers
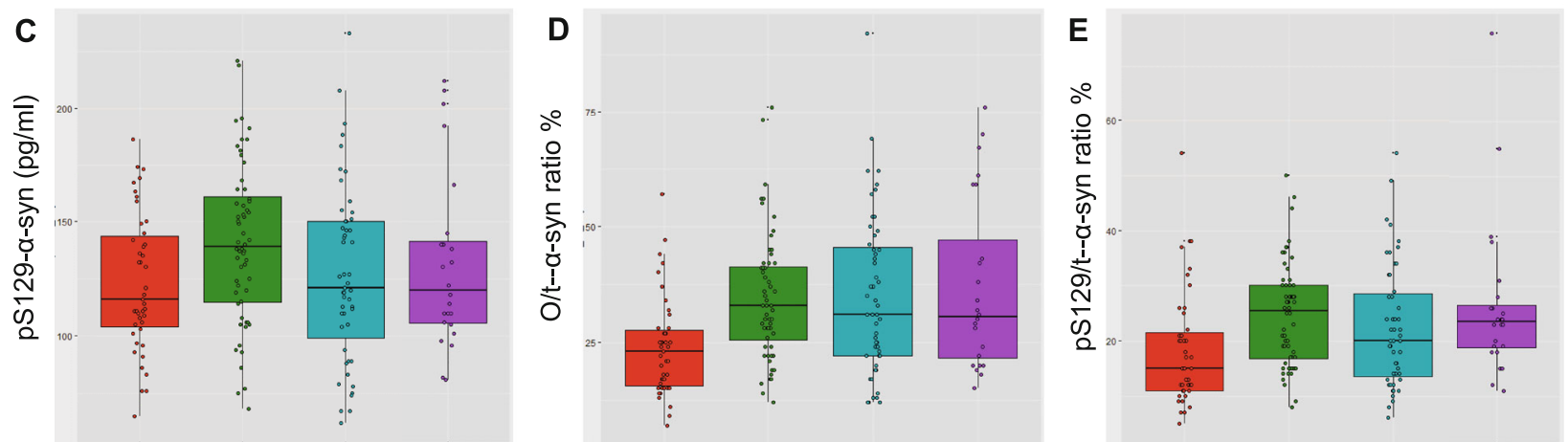

Fig. 1 Box-and-whiskers plots of CSF levels of a-syn forms in SPD, symptomatic and asymptomatic LRRK2 mutation carriers, and Ctrl. Box-andwhiskers plots of CSF levels of a-syn species in SPD, asymptomatic LRRK2 carriers, symptomatic LRRK2 carriers and Ctrls. a CSF levels of t-a-syn, $\mathbf{b}$ CSF levels of o- $a-5 y n, \mathbf{c}$ CSF levels of pSer129-a-syn, $\mathbf{d}$ ratio of o- $a-5 y n / t-a-s y n \%$, e ratio of pSer129-a-syn/ t-a-syn \%. The line through the middle of the boxes corresponds to the median and the lower and the upper lines to the 25th and 75th percentiles, respectively. The whiskers extend from the 5 th percentile on the bottom to the 95th percentile on top. Differences between groups were assessed with the GLM compared to Ctrl group and adjusted for age and gender. ${ }^{*} P<0.05,{ }^{*} P<0.01,{ }^{* *} P<0.001$

factors were assessed in CSF samples from all study participants. Of the 40 markers measured, 18 showed robust readings above background and were further analyzed (6Ckine, IL-6, SDF-1 $\alpha+\beta$, IL-16, MDC, MIF, TNF- $\alpha$, MPIF-1, Eotaxin, IP-10, MCP-1, IL-8, MCP-2, SCYP16, MIP-1Delta, CTACK, MIP-3 $\beta$, Fractalkine). GLM Bonferroni corrected analysis revealed that of the remaining 18 , only IL-16 and TNF- $\alpha$ were significantly different. A univariate, post-hoc analysis corrected for age and gender as covariates demonstrated that IL-16 levels were significantly lower in SPD group (median and IQR $=5.1 \quad(4-6.6) \mathrm{pg} / \mathrm{ml}, n=42)(P<0.05)$ compared with Ctrl (median and IQR $6.5=(4.94-8) \mathrm{pg} / \mathrm{ml}$, $n=28$ ). More interestingly, analysis showed a significant increase of TNF- $\alpha$ in asymptomatic LRRK2 mutation carriers (median and IQR $=5.5(4.2-7.4) \mathrm{pg} / \mathrm{ml}$ $(P<0.05), n=24)$ compared to Ctrl (median and IQR $=$ $3.3(2.6-5.5) \mathrm{pg} / \mathrm{ml}(P<0.05), n=28)$. The inflammatory profiles of symptomatic LRRK2 mutation carriers and SPD cases were not significantly different (data not shown).
By use of Spearman correlations, we evaluated associations between different CSF $\alpha$-syn forms and AD core biomarkers (Supplementary Table 1). For the Ctrl group, but not for any of the other groups, we found a positive association between o- $\alpha$-syn and pSer129- $\alpha$-syn $(r=$ $0.39, P<0.01)$. We also noted an inverse correlation between $\mathrm{t}$ - $\alpha$-syn and $\mathrm{o}-\alpha$-syn $(r=0.31, P<0.05)$ that was only present in asymptomatic LRRK2 mutation carriers. When we explored correlations between $\alpha$-syn species and the AD biomarkers, we found that $t-\alpha$-syn positively correlated with $\mathrm{A} \beta-40$ and $\mathrm{A} \beta-42$ in the asymptomatic LRRK2 group $(r=0.568, P<0.01, r=0.485, P<0.05)$. No other correlations were noted.

\section{Correlations between CSF alpha-synuclein levels and clinical parameters}

Correlational analyses of CSF levels of $\alpha$-syn species with clinical parameters (age, disease duration, UPDRS-III, H\&Y and MoCA) are shown in Supplementary Table 2. In summary, higher levels of CSF $t-\alpha$-syn correlated with: worse cognitive function as assessed by the 
Montreal Cognitive Assessment (MoCA) score $(r=-$ $0.44, P<0.01)$ in the sPD group. Similarly, a weak correlation of $\mathrm{t}-\alpha$-syn and age was noted in PD group $(r=$ $0.29, P<0.01)$. While pS129- $\alpha$-syn positively correlated with age in PD group $(r=0.384, P<0.01)$, aging was also associated with increased levels of CSF TNF- $\alpha$ in both $\mathrm{PD}$ and asymptomatic carriers groups $(r=0.362, P<0.01$, $r=0.492, P<0.01$, respectively).

\section{Discriminant function analysis}

In an attempt to identify the optimal panel that can serve as predictive markers for PD at the prodromal stage, we performed a discriminant function analysis of biomarkers that were significantly different between the groups. Canonical discriminant function classification results are presented in Table 2 . In the analysis, both groups of sPD and symptomatic LRRK2 carriers were combined as one PD group. A panel of $\mathrm{t}-$, $\mathrm{o}$ - and pS129$\alpha$-syn, TNF- $\alpha$ and IL- 16 together correctly classified $60 \%$ of all cases in the asymptomatic LRRK2 mutation carriers, PD, and Ctrl groups (lambda $=0.644, P<0.001$ ). The discrimination plot of the two canonical discriminant functions for discrimination of the three groups is presented in Fig. 2 and the loadings of individual predictors on each discriminant function are shown in Supplementary Table 3. Canonical discriminant function 1 strongly correlated with $\mathrm{t}-\alpha$-syn $\left(r=-0.694^{*}\right), \quad$ o- $\alpha$-syn $\left(r=-0.499^{*}\right)$ and $\mathrm{pS} 129-\alpha-\operatorname{syn}\left(r=0.390^{*}\right)$ and discriminated both asymptomatic LRRK2 mutation carriers and PD groups from Ctrl group; we will subsequently refer to this function as the Disease function. Canonical discriminant function 2 strongly correlated with TNF- $\alpha$ $\left(r=0.678^{*}\right)$ and IL-16 $\left(r=-0.554^{*}\right)$ and further discriminated asymptomatic LRRK2 mutation carriers group from Ctrl group; we will subsequently refer to this function as the Prodromal function. Asymptomatic LRRK2 mutation carriers' centroid is located at the intersection of both the Disease axis and the Prodromal disorders axis.

We employed backward-elimination multiple logistic regression analyses to identify optimal biomarker panels for bilateral comparisons between: [1] asymptomatic LRRK2 mutation carriers and Ctrl, and [2] PD patients and Ctrl, for which t-, o- and pS129- $\alpha$-syn, TNF- $\alpha$, and IL-16 were entered as predictors and the Ctrl group was used as the reference group in each comparison. A summary of the final models are shown in Table 3. The combination of $\mathrm{t}$ - $\alpha$-syn, o- $\alpha$-syn, and TNF- $\alpha$ discriminated the asymptomatic $L R R K 2$ mutation carriers group from the Ctrl group: low levels of $\mathrm{t}-\alpha$-syn (OR, 0.997; 95\% CI, 0.994-0.999), high levels of o- $\alpha$-syn (OR, 1.029, 95\% CI, 0.999-1.060), and high levels of TNF- $\alpha$ (OR, 1.418; 95\% CI, 0.998-2.014) indicate that individuals are at higher risk developing PD. Examining PD and Ctrl groups only, we found that low levels of $\mathrm{t}-\alpha$-syn (OR, 0.996; 95\% CI, 0.994-0.999), high levels of o- $\alpha$-syn (OR, 1.031; 95\% CI,1.005-1.056), high levels of pS129- $\alpha$-syn (OR, 1.035; 95\% CI, 1.010-1.059), low levels of IL-16 (OR, 0.785; 95\% CI, 0.603-1.022) differentiates the PD group from the Ctrl group. Receiver operating characteristic curves for both models are illustrated in Fig. 3. Each of the models generated an area under the curve (AUC) of $>0.80$.

\section{Discussion}

LRRK2 mutations are well-described as a cause of genetic familial parkinsonism, and drugs inhibiting LRRK2 kinase activity are already in clinical trials [24]. However, as $L R R K 2$ mutation carriers are at high risk of developing PD, asymptomatic individuals with $L R R K 2$ mutations are an excellent group for discovery of biomarkers of prodromal PD based on the premise that they are highly likely to develop PD in future.

In the current study, we measured 47 different candidate biomarkers and found evidence that low levels of CSF $\mathrm{t}-\alpha-$ syn, and high levels of CSF o- $\alpha$-syn and TNF- $\alpha$ potentially differentiate asymptomatic LRRK2 mutation carriers, i.e. PD subjects at the prodromal phase, from healthy controls. As these biomarkers in combination had greater discriminant power than those in isolation, these findings emphasize the value of combining multiple markers for early detection of PD.

In line with previous cross-sectional studies assessing the diagnostic power of CSF $\alpha$-syn forms $[8,9]$, we report that sPD patients had significantly decreased levels

Table 2 Canonical Discriminant functions classification results ${ }^{a}$

\begin{tabular}{|c|c|c|c|c|c|c|}
\hline \multirow[t]{2}{*}{ Group } & & & \multicolumn{3}{|c|}{ Predicted Group Membership } & \multirow[t]{2}{*}{ Total } \\
\hline & & & $\mathrm{ctrl}$ & PD & Asymptomatic carriers & \\
\hline \multirow[t]{6}{*}{ Original } & Count & Ctrl & 20 & 3 & 4 & 27 \\
\hline & & PD & 7 & 33 & 14 & 54 \\
\hline & & Asymptomatic carriers & 5 & 9 & 10 & 24 \\
\hline & $\%$ & Ctrl & 74.1 & 11.1 & 14.8 & 100.0 \\
\hline & & PD & 13.0 & 61.1 & 25.9 & 100.0 \\
\hline & & Asymptomatic carriers & 20.8 & 37.5 & 41.7 & 100.0 \\
\hline
\end{tabular}

\footnotetext{
a $60.0 \%$ of original grouped cases correctly classified
} 


\section{Canonical Discriminant Functions}

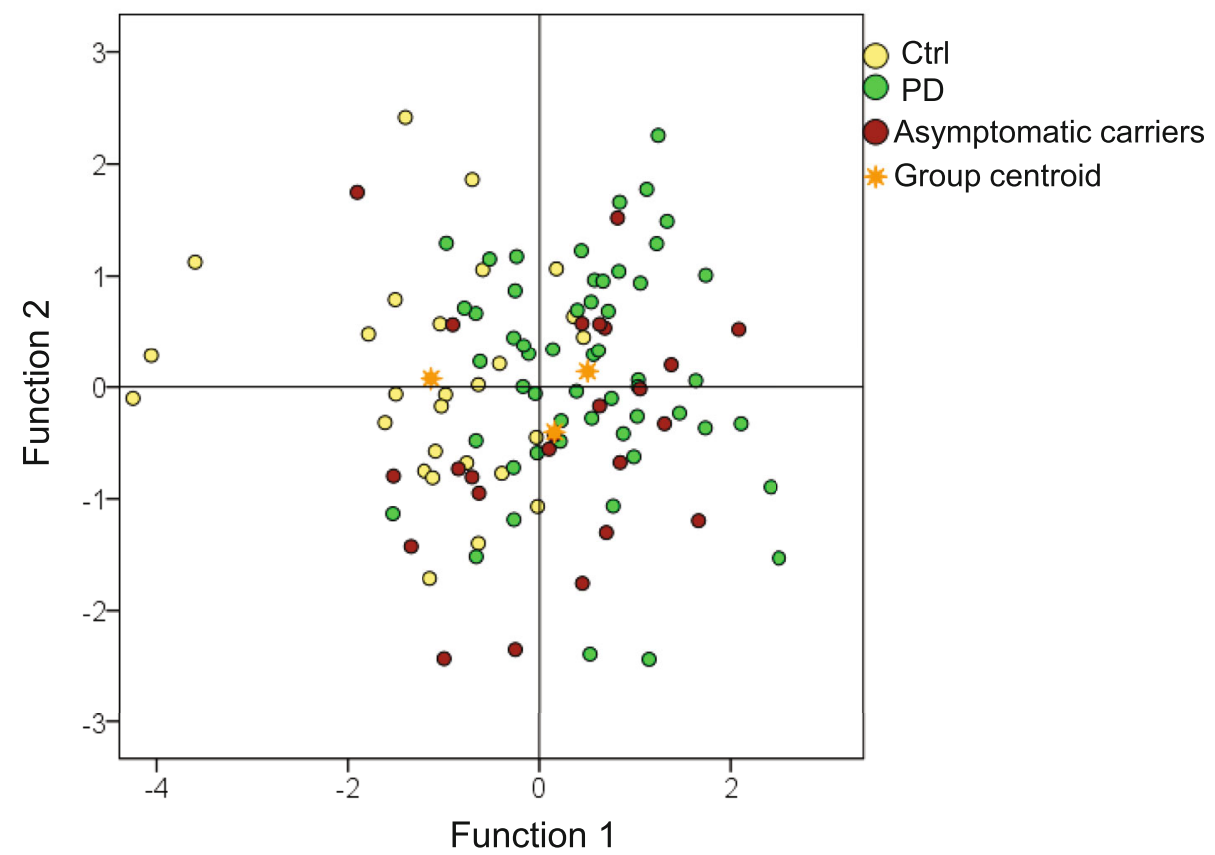

Fig. 2 Discriminant function plot of canonical discriminant functions. CSF biomarkers were z-transformed before analyses. Discriminant function plot of canonical discriminant functions for discrimination of asymptomatic LRRK2 mutation carriers, PD and controls. Yellow dots indicate individual data of control subjects, green dots indicate individual data of Parkinson's disease patients (sporadic and symptomatic LRRK2 mutation carriers) and red dots indicate individual data of LRRK2 asymptomatic carriers. The golden stars represent the group centroids

of CSF t- $\alpha$-syn and increased levels of CSF o- and pS129- $\alpha$-syn compared to Ctrl group. Importantly, our results also show that asymptomatic LRRK2 mutation carriers displayed significantly higher CSF o- $\alpha$-syn than Ctrls. These findings support the hypothesis that $\alpha$-syn oligomerization is an early event in the pathophysiology of PD and add further weight to evaluation of CSF o- $\alpha$ syn as a candidate biomarker for detection of prodromal PD. A previous study showed a similar trend, although the results were not significant [16]. One possible explanation for disparate results between both studies is that the use of conformation-specific antibodies and oligomeric-specific ELISA in the present study, minimized the overlap between groups and improved the discriminant power between the study groups.

Our results partially contradict with Vilas et al., 2016 study, where CSF $t-\alpha$-syn levels were lowered only in idiopathic (i.e. sporadic) PD group in contrast to other diagnostic groups; however, the differences in CSF tTau, pTau, $A \beta-40$ or $A \beta-42$ levels didn't reach statistical significance. Immunoassays employed to measure $A D$ biomarkers in both studies used Luminex xMAP but employed different antibodies, which may underlie different results. If this is the case, this would have important implications for the design of future biomarkers studies.

Table 3 Logistic regression analysis of multiple CSF biomarkers

\begin{tabular}{|c|c|c|c|c|}
\hline \multicolumn{5}{|l|}{ Ctrl Group } \\
\hline \multicolumn{5}{|l|}{ Choup } \\
\hline Asymptomatic carriers & $\begin{array}{l}\text { t-a-syn } \\
\text { o-a-syn } \\
\text { TNF-a }\end{array}$ & $\begin{array}{l}0.997(0.994-0.999) \\
1.029(0.999-1.060) \\
1.418(0.998-2.014)\end{array}$ & $\begin{array}{l}\text { AUC: } 0.843(0.724-0.961) \\
\text { Sens: } 87.5 \% \\
\text { Spec: } 66.7 \%\end{array}$ & 0.000 \\
\hline PD (i.e. sPD \& symptomatic LRRK2 mutation carriers) & $\begin{array}{l}\text { t-a-syn } \\
\text { o-a-syn } \\
\text { pS129-a-syn } \\
\text { IL-16 }\end{array}$ & $\begin{array}{l}0.996(0.994-0.999) \\
1.031(1.005-1.056) \\
1.035(1.010-1.059) \\
0.785(0.60-1.022)\end{array}$ & $\begin{array}{l}\text { AUC: } 0.896(0.823-0.969) \\
\text { Sens: } 87.0 \% \\
\text { Spec: } 78.6 \%\end{array}$ & 0.000 \\
\hline
\end{tabular}

AUC area under the curve, $H C$ Healthy controls, NPV negative predictive value, OR odds ratio, o-a-syn oligomeric a-synuclein, $P D$ sporadic and symptomatic $L R R K 2$ mutation patients, PPV positive predictive value; pSer129-a-synuclein, phosphorylated a-synuclein protein at serine 129, Sens sensitivity, Spec specificity, TNF-a tumor necrosis factor-alpha, $t$-a-syn total a-synuclein 

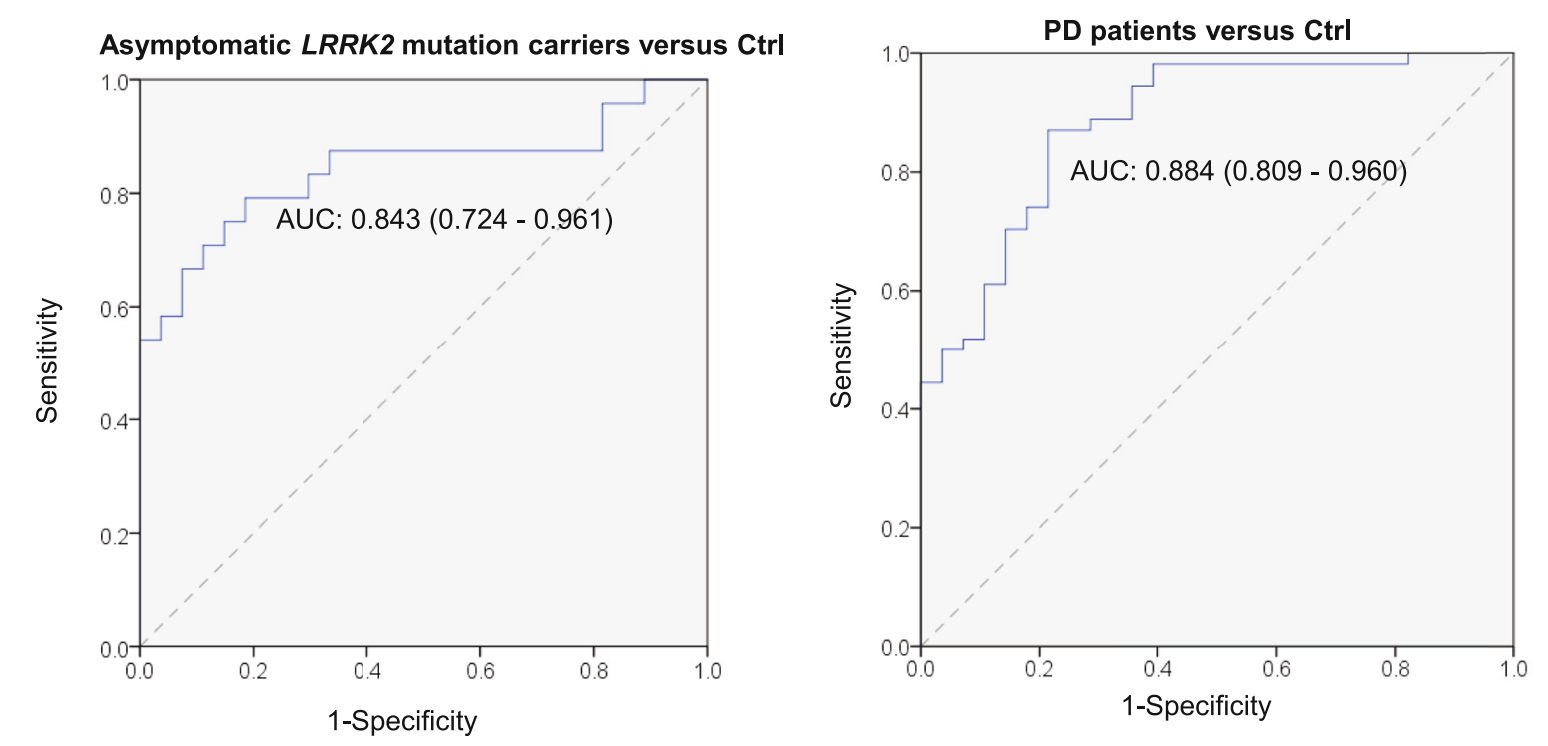

Fig. 3 Receiver operating characteristic curves (ROC) showing the diagnostic accuracy of the final logistic regression models. Asymptomatic LRRK2 mutation carriers vs Ctrl subjects, (B) PD patients vs Ctrl subjects

Several biomarkers and brain tissue studies have shown that neuroinflammatory process precedes neurodegeneration in PD [25-27], whilst very few studies have evaluated inflammatory biomarkers in relation to $\alpha$-syn forms [15]. In the current study, we found CSF TNF- $\alpha$ to be significantly higher in asymptomatic LRRK2 mutation carriers compared to Ctrls. In a recent 11-year study in over 4 million Norwegians, an inhaled asthma medication with anti-TNF activity, a brain-penetrant drug, was associated with lower levels of $t-\alpha$-syn and decreased the risk of PD $[28,29]$. While the authors of the study didn't claim a causative association, their findings correspond with our present findings, as asymptomatic LRRK2 mutation carriers showed significantly lower CSF $\mathrm{t}-\alpha$-syn and higher TNF- $\alpha$ and o- $\alpha$-syn. However, the control subjects in our study were significantly younger than sPD, symptomatic and asymptomatic LRRK2 mutation carriers $(p<0.05)$, a notable finding given that previous studies have demonstrated an age-dependent increase in CSF TNF- $\alpha$ levels [30], which we also observed (Supplementary Table 2). However, CSF TNF- $\alpha$ levels were still significantly increased in asymptomatic LRRK2 mutation carriers compared with Ctrl subjects when CSF TNF- $\alpha$ values were analyzed after age adjustment (Table 1). We observed no effect of gender on biomarker levels in all study groups, and analysis was performed with and without gender adjustment with no difference in overall findings. Therefore, we concluded that gender bias did not affect the results in this study.

It is important to note that LRRK2 mutations (G2019S, N1437H) have incomplete penetrance, meaning that not all of the asymptomatic LRRK2 mutation carriers in our cohort would develop PD. This may explain the overlap between asymptomatic LRRK2 mutation carriers and both Ctrl and sPD groups (Fig. 1), falling almost at the intersection in the discriminant function analysis (Fig. 3). In light of the incomplete penetrance of $L R R K 2$, discriminant function analysis correctly classified $60.0 \%$ of original grouped cases, where $74.1 \%$ Ctrl, $61.1 \%$ PD and $41.7 \%$ cases of asymptomatic LRRK2 mutation carriers were correctly classified (Table 2). It is tempting to speculate that the lower discriminant function analysis levels for asymptomatic LRRK2 cases reflects the incomplete penetrance of this mutation, and could suggest that the cases correctly discriminated are those most likely to develop PD. Future studies could further explore this question in LRRK2 patients in large prospective studies, such as the ongoing Parkinson's progression markers initiative, to determine their diagnostic utility in predicting PD in LRRK2 mutation carriers.

In a recent study by Halliday and colleagues, using CSF and serum samples from the Michael J. Fox Foundation LRRK2 cohort consortium [15], 28 cytokines were measured in CSF and compared between Ctrl $(n=22)$ and asymptomatic LRRK2 mutation carriers $(n=25)$. However, in this study, none of the markers, including TNF- $\alpha$, distinguished between the two groups but CSF TNF- $\alpha$ levels combined with 5 other cytokines significantly differentiated these cases from sPD $(n=29)$ and symptomatic LRRK2 mutation (G2019S) carriers. The discrepancy seen in our results and the above mentioned study could be due to the differences in the inclusion and exclusion 
criteria of the subjects, and/or time difference in processing or storing the samples.

Admittedly, the size of our cohort is relatively small, particularly considering the number of subjects in which all the biomarkers' measurements were available. Furthermore, the only control group in this study was composed of first-degree relatives of LRRK2 mutations carriers and, although they were confirmed negative for LRRK2 mutations, including another standard control group shall be considered in further studies. Previous studies have described heterogeneous pathologies in LRRK2 mutation carriers, where different brain areas and methods were studied and used [31-33]. Such differences in neuropathological changes elicited by LRRK2 mutations necessitates the need for cohorts where neuropathological examination of LRRK2 mutation carriers is conducted both to provide neuropathological confirmation of clinical diagnoses, and to better define and validate differential profiles of biomarkers.

\section{Conclusions}

Our study, by demonstrating lower CSF t- $\alpha$-syn levels, higher o- $\alpha$-syn and TNF- $\alpha$ in asymptomatic LRRK2 mutation carriers highlights the power of those biomarkers at providing an early detection of PD. Future studies are necessary to confirm the potential sensitivity and specificity of combing $\alpha$-syn species with inflammatory biomarkers as predictive and perhaps progression biomarkers of $\mathrm{PD}$. Comparison of CSF and blood levels of the current biomarkers in larger cohorts with longitudinal follow-up of LRRK2 mutation carriers, and other "at risk" groups, are of great importance. Such studies are crucial to select biomarkers that could identify individuals at high risk to convert to $\mathrm{PD}$, who would then be the target group for the development of preventive treatments.

\section{Supplementary information}

Supplementary information accompanies this paper at https://doi.org/10. 1186/s40035-020-00192-4.

Additional file 1: Supplementary Table 1. Associations between CSF biomarkers. No correlation between CSF biomarkers were present in symptomatic LRRK2 mutation carriers group alone. Both SPD and symptomatic LRRK2 mutation carriers groups were combined as one PD group. Associations between CSF biomarkers were assessed with Spearman correlation coefficients. Data shown as r. Significance: ${ }^{* *} p<$ 0.001 ; ${ }^{* *} p<0.01$; ${ }^{*} p<0.05 \mathrm{~A} \beta 1-42$, amyloid $\beta 1-42$; Ctrl, Healthy controls; o- $a$-syn, oligomeric a-synuclein; pSer129-a-synuclein, phosphorylated asynuclein protein at serine 129; pTau, tau phosphorylated at threonine 181; PD, Parkinson's disease patients; tTau, total tau protein; and t-a-syn, total a-synuclein. Supplementary Table 2. Associations between CSF asyn species and clinical parameters. Associations between CSF biomarkers were assessed with Spearman correlation coefficients. Data shown as $r$. Significance: ${ }^{* *} p<0.001$; ${ }^{* *} p<0.01 ; * p<0.05$. Both sPD and symptomatic LRRK2 mutation carriers groups were combined as one PD group. $A \beta 1-42$, amyloid $\beta 1-42 ;$ Ctrl, healthy controls; H\&Y, Hoehn and Yahr scale; MoCA, Montreal Cognitive Assessment; NA, not applicable; o-a-syn, oligomeric a-synuclein; pSer129-a-synuclein, phosphorylated a-synuclein protein at serine 129; pTau, tau phosphorylated at threonine 181; sPD, sporadic PD; TNF-a, tumor necrosis factor-alpha; tTau, total tau protein; ta-syn, total a-synuclein; UPDRS-III, Unified Parkinson's Disease Rating Scale. Supplementary Table 3. Discriminant loadings for each individual predictor. The correlation coefficient represents the relative contribution for each predictor to group separation. IL-16, interlukin-16; o-a-syn, a-synuclein oligomers; pS129-a-syn, phosphorylated Ser 129 a-synuclein; t-a-syn, total a-synuclein; TNF- a, tumor necrosis factor- $a$.

\section{Abbreviations}

PD: Parkinson's disease; CSF: Cerebrospinal fluid; TNF-a: Tumor necrosis factor-alpha; AUC: Area under the curve; PET: Positron emission tomography; AD: Alzheimer's disease; UPDRS: Unified Parkinson's disease rating scale; MAO: Monoamine oxidase inhibitor; PPMI: Parkinson's Progression Markers Initiative; ELISA: Enzyme Linked Immunosorbent Assay; PBS: Phosphatebuffered saline; PBS-T: Phosphate-buffered saline with tween-20; HRP: Horseradish peroxidase; HC: Healthy control; OR: Odds ratio; Cl: Confidence interval; GLM: General linear model; IL: Interleukin; IQR: Interquartile range; NPRP: National Priorities Research Program

\section{Acknowledgments}

The authors thank all patients and healthy subjects, lab manager Dr. Houari Abdesselem, funding agencies, Michael J Fox Foundation and Norwegian Parkinson Foundation This work would have not been possible without their valuable contribution.

\section{Disclosures}

The authors have no financial disclosures or conflicts of interest to report.

\section{Authors' contributions}

Study design and supervision: N.M, O.E. Conduction of the experiments: N.M, M.T. and N.V. Clinical data collection: J.A. and E.H. Data analysis: N.M. and N.E. Writing the first draft of the manuscript and incorporating revisions from other authors: N.M. Data interpretation and critical revision of the manuscript for important intellectual content: N.M, J.A., W.B., T.T., B.M., H.B., and O.E. Final review of the draft and approval to submit for publication: O.E. All authors read and approved the final manuscript.

\section{Funding}

This study was supported by Strat-up Funding to OE from Qatar Biomedical Research Institute (SF 2007-007) and Qatar National Research Fund (NPRP No.: 8-517-3-112)

\section{Availability of data and materials}

The datasets used and/or analyzed during the current study are available from the corresponding author on reasonable request.

\section{Ethics approval and consent to participate}

All patients provided signed informed consent, and the study was approved by the Regional Committee for Medical and Health Research Ethics.

\section{Consent for publication}

Not applicable.

\section{Competing interests}

The authors declare that they have no competing interests.

\section{Author details}

${ }^{1}$ Neurological Disorders Research Center, Qatar Biomedical Research Institute, Hamad Bin Khalifa University, Qatar Foundation, P.O. Box 5825, Doha, Qatar.

${ }^{2}$ Department of Neuroscience, Norwegian University of Science and

Technology, (NTNU), Trondheim, Norway. ${ }^{3}$ Department of Neurology, St. Olav's Hospital, University Hospital of Trondheim, Trondheim, Norway. ${ }^{4}$ Clinical Epidemiology, Sidra Medical and Research Center, Doha, Qatar. ${ }^{5}$ Department of Anatomy and Neurosciences, Neuroscience Campus Amsterdam, VU University Medical Centre, Amsterdam, the Netherlands. ${ }^{6}$ Department of Neurology, Research Institute for Geriatrics, Kyoto Prefectural University of Medicine, Kyoto 602-0841, Japan. ${ }^{7}$ Paracelsus-Elena-Klinik, Klinikstraße, Kassel, and University Medical Center Göttingen, Department of 
Neurology, Göttingen, Germany. ${ }^{8}$ Department of Neurology, Amsterdam UMC, location VU University Medical Centre, Amsterdam, The Netherlands.

Received: 20 January 2020 Accepted: 17 April 2020

Published online: 06 May 2020

\section{References}

1. Wu YR, Chang KH, Chang WT, Hsiao YC, Hsu HC, Jiang PR, et al. Genetic variants of LRRK2 in Taiwanese Parkinson's disease. PLoS One. 2013:8(12): e82001.

2. Moehle MS, Webber PJ, Tse T, Sukar N, Standaert DG, DeSilva TM, et al. LRRK2 inhibition attenuates microglial inflammatory responses. J Neurosci. 2012;32(5):1602-11.

3. Dzamko N, Halliday GM. An emerging role for LRRK2 in the immune system. Biochem Soc Trans. 2012;40(5):1134-9.

4. Dzamko N, Geczy CL, Halliday GM. Inflammation is genetically implicated in Parkinson's disease. Neuroscience. 2015;302:89-102.

5. Hawkes $\mathrm{CH}$, Del Tredici K, Braak H. Parkinson's disease: a dual-hit hypothesis. Neuropathol Appl Neurobiol. 2007:33(6):599-614.

6. Stewart T, Sossi V, Aasly JO, Wszolek ZK, Uitti RJ, Hasegawa K, et al. Phosphorylated a-synuclein in Parkinson's disease: correlation depends on disease severity. Acta Neuropathol Commun. 2015;3:7.

7. Tokuda T, Qureshi MM, Ardah MT, Varghese S, Shehab SA, Kasai T, et al. Detection of elevated levels of a-synuclein oligomers in CSF from patients with Parkinson disease. Neurology. 2010;75(20):1766-72.

8. Park MJ, Cheon SM, Bae HR, Kim SH, Kim JW. Elevated levels of a-synuclein oligomer in the cerebrospinal fluid of drug-naïve patients with Parkinson's disease. J Clin Neurol. 2011;7(4):215-22.

9. Majbour NK, Vaikath NN, van Dijk KD, Ardah MT, Varghese S, Vesterager LB, et al. Oligomeric and phosphorylated alpha-synuclein as potential CSF biomarkers for Parkinson's disease. Mol Neurodegener. 2016:11(1):7.

10. Compta Y, Martí MJ, Ibarretxe-Bilbao N, Junqué C, Valldeoriola F, Muñoz E, et al. Cerebrospinal tau, phospho-tau, and beta-amyloid and neuropsychological functions in Parkinson's disease. Mov Disord. 2009; 24(15):2203-10.

11. Parnetti L, Gaetani L, Eusebi P, Paciotti S, Hansson O, El-Agnaf O, et al. CSF and blood biomarkers for Parkinson's disease. Lancet Neurol. 2019:18(6): 573-86.

12. van Steenoven I, Majbour NK, Vaikath NN, Berendse HW, van der Flier WM, van de Berg WDJ, et al. a-Synuclein species as potential cerebrospinal fluid biomarkers for dementia with lewy bodies. Mov Disord. 2018;33(11):172433.

13. Poewe W. Parkinson's disease and the quest for preclinical diagnosis: an interview with professor Werner Poewe. Neurodegener Dis Manag. 2017; $7(5): 273-7$.

14. Vilas D, Shaw LM, Taylor P, Berg D, Brockmann K, Aasly J, et al. Cerebrospinal fluid biomarkers and clinical features in leucine-rich repeat kinase 2 (LRRK2) mutation carriers. Mov Disord. 2016:31(6):906-14.

15. Dzamko N, Rowe DB, Halliday GM. Increased peripheral inflammation in asymptomatic leucine-rich repeat kinase 2 mutation carriers. Mov Disord. 2016;31(6):889-97.

16. Aasly JO, Johansen KK, Brønstad G, Warø BJ, Majbour NK, Varghese S, et al. Elevated levels of cerebrospinal fluid a-synuclein oligomers in healthy asymptomatic LRRK2 mutation carriers. Front Aging Neurosci. 2014;6:248

17. Aasly JO, Toft M, Fernandez-Mata I, Kachergus J, Hulihan M, White LR, et al. Clinical features of LRRK2-associated Parkinson's disease in Central Norway. Ann Neurol. 2005;57(5):762-5.

18. Aasly JO, Vilariño-Güell C, Dachsel JC, Webber PJ, West AB, Haugarvoll K, et al. Novel pathogenic LRRK2 p.Asn1437His substitution in familial Parkinson's disease. Mov Disord. 2010;25(13):2156-63.

19. Johansen KK, Hasselberg K, White LR, Farrer MJ, Aasly JO. Genealogical studies in LRRK2-associated Parkinson's disease in Central Norway. Parkinsonism Relat Disord. 2010;16(8):527-30.

20. Gelb DJ, Oliver E, Gilman S. Diagnostic criteria for Parkinson disease. Arch Neurol. 1999;56(1):33-9.

21. Johansen KK, White LR, Farrer MJ, Aasly JO. Subclinical signs in LRRK2 mutation carriers. Parkinsonism Relat Disord. 2011;17(7):528-32.

22. Vaikath NN, Majbour NK, Paleologou KE, Ardah MT, van Dam E, van de Berg $W D$, et al. Generation and characterization of novel conformation-specific monoclonal antibodies for a-synuclein pathology. Neurobiol Dis. 2015;79: 81-99.
23. Majbour NK, Vaikath NN, Eusebi P, Chiasserini D, Ardah M, Varghese S, et al. Longitudinal changes in CSF alpha-synuclein species reflect Parkinson's disease progression. Mov Disord. 2016;31(10):1535-42.

24. Tolosa E, Vila M, Klein C, Rascol O. LRRK2 in Parkinson disease: challenges of clinical trials. Nat Rev Neurol. 2020;16(2):97-107.

25. Ferger B, Leng A, Mura A, Hengerer B, Feldon J. Genetic ablation of tumor necrosis factor-alpha (TNF-alpha) and pharmacological inhibition of TNFsynthesis attenuates MPTP toxicity in mouse striatum. J Neurochem. 2004; 89(4):822-33.

26. Bartels AL, Leenders KL. Neuroinflammation in the pathophysiology of Parkinson's disease: evidence from animal models to human in vivo studies with [11C]-PK11195 PET. Mov Disord. 2007;22(13):1852-6.

27. Cebrián C, Zucca FA, Mauri P, Steinbeck JA, Studer L, Scherzer CR, et al. MHC-I expression renders catecholaminergic neurons susceptible to T-cellmediated degeneration. Nat Commun. 2014;5:3633.

28. Mittal S, Bjørnevik K, Im DS, Flierl A, Dong X, Locascio JJ, et al. $\beta 2$ Adrenoreceptor is a regulator of the $a$-synuclein gene driving risk of Parkinson's disease. Science. 2017;357(6354):891-8.

29. Clark IA, Vissel B. Therapeutic implications of how TNF links apolipoprotein E, phosphorylated tau, $\alpha$-synuclein, amyloid- $\beta$ and insulin resistance in neurodegenerative diseases. Br J Pharmacol. 2018;175(20):3859-75.

30. Bruunsgaard H, Skinhøj P, Pedersen AN, Schroll M, Pedersen BK. Ageing, tumour necrosis factor-alpha (TNF-alpha) and atherosclerosis. Clin Exp Immunol. 2000;121(2):255-60.

31. Henderson MX, Sengupta M, Trojanowski JQ, Lee VMY. Alzheimer's disease tau is a prominent pathology in LRRK2 Parkinson's disease. Acta Neuropathol Commun. 2019;7(1):183.

32. Poulopoulos M, Levy OA, Alcalay RN. The neuropathology of genetic Parkinson's disease. Mov Disord. 2012;27(7):831-42.

33. Kalia LV, Lang AE, Hazrati LN, Fujioka S, Wszolek ZK, Dickson DW, et al. Clinical correlations with Lewy body pathology in LRRK2-related Parkinson disease. JAMA Neurol. 2015;72(1):100-5
Ready to submit your research? Choose BMC and benefit from:

- fast, convenient online submission

- thorough peer review by experienced researchers in your field

- rapid publication on acceptance

- support for research data, including large and complex data types

- gold Open Access which fosters wider collaboration and increased citations

- maximum visibility for your research: over $100 \mathrm{M}$ website views per year

At BMC, research is always in progress.

Learn more biomedcentral.com/submissions 